\title{
Propuesta de laboratorio de certificación en la norma ISO 29119 de pruebas de calidad de software en el Centro de Servicios y Gestión Empresarial del SENA
}

\section{Proposal for a certified laboratory in the iso 29119 standard for software quality tests in the SENA Center for Services and Business Management}

\author{
Ricardo Gómez Giraldo a \& Lina María Jaramillo Terán b \\ a Investigador Sennova, Centro de Servicio y Gestión Empresarial, SENA, GIGAT, Medellín, Colombia, \\ rgomez10@misena.edu.co \\ b Instructora, Centro de Servicio y Gestión Empresarial, SENA, GIGAT, Medellín, Colombia, linajate@gmail.com
}

Recibido: diciembre 6, 2019. Aceptado: diciembre 26, 2019.

\begin{abstract}
Resumen
Este trabajo propone la estructuración de un laboratorio de pruebas de calidad de software bajo el lineamiento de la norma ISO 29119 con el objetivo de certificar la calidad de los productos desarrollados por la Fábrica de Software de una entidad de formación o clientes externos. Una revisión bibliográfica permitió identificar los diferentes procesos y técnicas de pruebas, a su vez los diferentes formatos de apoyo para registrar su trazabilidad. Finalmente, se establece el procedimiento operativo para el funcionamiento y la certificación de un laboratorio de pruebas de software en el Centro de Servicios y Gestión Empresarial del SENA Regional Antioquia
\end{abstract}

Palabras Clave: Servicios Tecnológicos, Laboratorio para pruebas, calidad de software, prueba de software, ISO 29919.

\begin{abstract}
This work proposes the structuring of a software quality testing laboratory under the guidelines of ISO 29119 intending to certify the quality of the products generated by the Software development team of a training entity or external clients. A bibliographic review allowed for the identification of the different processes and testing techniques, and the required documentation formats to record their traceability. Finally, the paper establishes the operating procedure for the operation and certification of a software testing laboratory in the Business Management and Services Center of the SENA Regional Antioquia.
\end{abstract}

Keywords: Technological Services, Laboratory for testing, software quality, software testing, ISO 29919

\section{INTRODUCCIÓN}

Colombia, como país con una economía en búsqueda del crecimiento tecnológico, ha incursionado en la industria de desarrollo de software, aunque presentando un crecimiento moderado y por debajo de lo esperado. En Colombia las empresas de desarrollo de software carecen de preparación para ser competitivas internacionalmente; esto es válido para Latinoamérica en general, donde el sector informático se enfrenta a una serie de problemas como la dependencia tecnológica, el desconocimiento de la importancia que tiene el proceso de desarrollo sobre la calidad del producto y la construcción de software de forma artesanal, empírica y caótica [1]. Como industria, el software requiere de productos y servicios de alta calidad, lo cual se logra mediante la aplicación de modelos y metodologías de reconocidos internacionalmente [2] y una estrategia clave para el éxito de esta industria es certificar la calidad de procesos y producto [3], [4].

Salazar, Lizano y Lara definen las pruebas de software como la "verificación dinámica del comportamiento de un determinado elemento de software, utilizando un conjunto finito de casos de prueba, seleccionados a partir de un conjunto usualmente infinito de posibilidades de ejecución de ese elemento de software, para verificar el comportamiento esperado buscando minimizar la cantidad de errores" [5]. A su vez, el uso de software validado permite disminuir errores y bajar los costos de desarrollo, aumentando la confianza y

\section{Citar como:}

Ricardo Gómez Giraldo \& Lina María Jaramillo Terán. "Propuesta de laboratorio de certificación en la norma ISO 29119 de pruebas de calidad de software en el Centro de Servicios y Gestión Empresarial del SENA". Revista CINTEX, Vol. 24(2), pp. 46-54. 2019. 
seguridad en la aplicación del software [6]. Se estima, incluso, que entre el $50 \%$ y el $60 \%$ del esfuerzo total de producir software se invierte en tareas de aseguramiento de calidad [7], teniendo en cuenta que las pruebas deben realizarse bajo normatividad aceptada internacionalmente [8], [9] pero también considerando que la elección de las normas debe realizarse según las necesidades particulares de cada organización [10], [11], así como la metodología de diseño [12].

Teniendo en cuenta lo anterior, actualmente en el Centro de Servicios y Gestión Empresarial (CESGE) del SENA Regional Antioquia se dispone de infraestructura para la prestación de servicios de desarrollo de software que contribuye a la formación especializada de los aprendices [13], donde se crean condiciones de competitividad que al articularse con el sector productivo permite que los futuros egresados tengan un perfil más acorde a las necesidades de la industria.

Como lo menciona Fandos Garrido (2005), los centros de producción tienen como característica primordial realizar labores de tipo económico y generar utilidades para que éstas puedan ser destinadas a otras actividades del quehacer académico sin dejar de lado la investigación y la formación profesional, factores que permitirán ofertar productos con valor agregado [14]. EI SENA ofrece servicios tecnológicos a clientes Sena y externos con gran demanda para lo cual requiere contar con la certificación de calidad de dichos productos.

Por lo anterior, el CESGE promueve la creación de un laboratorio de pruebas de calidad de software que permiten trabajar con proyectos reales del sector empresarial articulado con la Fábrica de software bajo el lineamiento de la norma ISO 29119; lo cual no solo contribuirá a los procesos formativos de los aprendices del Sena de los programas relacionados con el desarrollo de software, sino que también impactará al sector empresarial ofreciéndoles por medio de este laboratorio un portafolio que entregue el apoyo necesario y suficiente a los empresarios e innovadores para la validación de sus desarrollos.

\section{METODOLOGÍA}

\subsection{La norma ISO 29119 como lineamiento en la definición del proceso de prueba}

Se ha reportado que la norma ISO/IEC 29119 proporciona a las pequeñas empresas de desarrollo de software un camino claro para implementar procesos sistemáticos cubriendo los procesos estratégicos, misionales y de apoyo, de acuerdo con los resultados obtenidos con cuatro compañías españolas y polacas [15]. Cabe resaltar que no basta con la implementación del laboratorio de pruebas para ofrecer los servicios que garanticen la calidad en los productos de software, es necesario encontrar un modelo que otorgue el lineamiento para la definición de los procesos.

Según lo anterior, existen casos de implementación donde se indica que la norma ISO 29119 es el instrumento que han utilizado como referente internacional en el ámbito de las pruebas; por ende, se define que el laboratorio se basará en la norma en mención con el propósito de generar criterios enfocados a determinar y asegurar la calidad de software durante todo el ciclo de vida del producto.

\subsection{Estructuración de los procesos del laboratorio}

Para el desarrollo de la estructuración de los procesos del laboratorio de pruebas se analizaron cada una de las partes de la norma ISO 29119:

- Parte uno: Conceptos y definiciones

- Parte dos: Modelo de proceso de pruebas

- Parte tres: Documentación de pruebas

- Parte cuatro: Técnicas de pruebas

En la parte uno de conceptos y definiciones la norma explica la importancia de las pruebas de software en las organizaciones, los procesos de pruebas en los ciclos de vida detallando cada ciclo con una visión muy general, las pruebas basadas en los riesgos donde el tester tiene el criterio para definir qué artefactos son más críticos que otros, rigiéndose por los requisitos, las estrategias de prueba acerca de la manera en que estas actividades están secuenciadas y organizadas en la planificación.

En la parte dos del modelo del proceso de pruebas la norma explica la planificación, el control, el seguimiento y la finalización de las pruebas, incluyendo el diseño e implementación, gestión del entorno, ejecución y reporte de incidencias.

En la parte tres de la documentación de las pruebas la norma define las plantillas que pueden ser utilizadas para la documentación de los productos de la parte dos

En la prte cuatro la norma define las técnicas que pueden ser usadas para el diseño de los casos de pruebas las cuales pueden ser basadas en las especificaciones, en la estructura y en la experiencia 
Propuesta de laboratorio de certificación en la norma ISO 29119 de pruebas de calidad de software en el Centro de Servicios

y Gestión Empresarial del SENA

Proposal for a certified laboratory in the iso 29119 standard for software quality tests in the SENA Center for Services and

De acuerdo en cada una de las partes antes mencionadas, se definieron las siguientes fases que fueron concertadas con el líder del proceso de la organización:

- Fase uno: Análisis de los términos de conceptos y definiciones sobre pruebas de software y artículos relacionados con pruebas de software.

- Fase dos: Análisis y diseño del proceso de pruebas del laboratorio.

- Fase tres: Se definen la política y la estrategia de pruebas para la organización, el objetivo, el alcance y los responsables del laboratorio, a su vez se realizó el diseño de las plantillas de entrada y salida del proceso de pruebas.

- Fase cuatro: Se definen las técnicas para la elaboración de los casos pruebas y se finaliza con el diseño de los reportes del proceso.

\section{RESULTADOS}

A continuación, se exponen como fueron desarrolladas las fases del proyecto que tiene como resultado principal la definición del proceso de prueba de calidad de software en el Centro de Servicios y Gestión Empresarial SENA.

El proceso de prueba consiste en la planificación, análisis y diseño de material de prueba, ejecución y registro de la prueba incluyendo el registro de las incidencias y finalización de la prueba e informes. El proceso permitirá evaluar la funcionalidad de un software de manera organizada especialmente aquellas que tienen que ver con el diseño y ejecución de las pruebas. Para poder definir el proceso de prueba es necesario conocer la estructura de roles la cual se muestra en la figura 1.

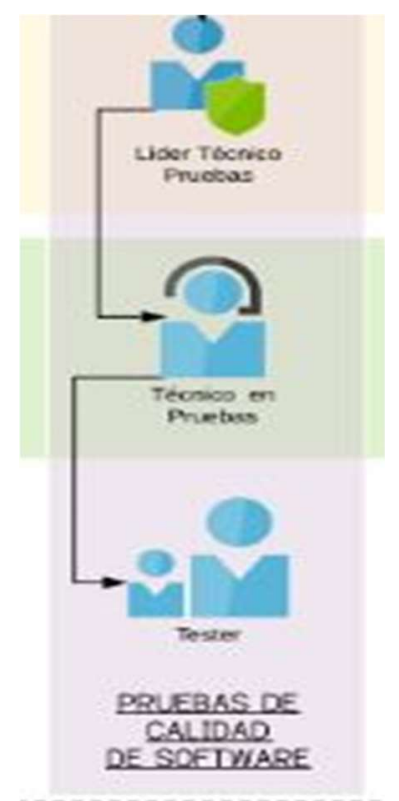

Figura 1. Estructura de roles laboratorio de pruebas

\subsection{ESTRUCTURA DE ROLES LABORATORIO DE PRUEBAS}

- Líder laboratorio de pruebas: Es el encargado de velar porque todas las tareas que se ejecuten en el laboratorio de pruebas se lleven a cabo de la manera más eficiente posible, aprovechando el tiempo y los recursos disponibles

- Auxiliar laboratorio de pruebas: Es el encargado de garantizar que el entorno para las pruebas este adecuado correspondiente a la infraestructura y el software.

- Tester: Es el encargado de probar la aplicación ejecutando los casos de prueba definidos en el equipo de proyecto. A su vez registra los incidentes detectados durante la prueba en el formato definido para ello. 


\subsection{FASES DEL PROYECTO \\ 3.2.1 FASE UNO}

En la tabla 1 se relacionan conceptos relacionados con las pruebas de software lo que permitió concluir que las pruebas de calidad de software son técnicas que cumplen el objetivo de identificar defectos en el software construido y para ello utiliza los requerimientos del cliente asegurando que se cumplan.

TABLA 1

CONCEPTOS Y ATRIBUTOS RELACIONADOS CON LAS PRUEBAS DE SOFTWARE

\begin{tabular}{|c|c|}
\hline Concepto & Definición \\
\hline $\begin{array}{l}\text { Prueba } \\
\text { Software }\end{array}$ & $\begin{array}{l}\text { de Las pruebas de software son las investigaciones empíricas y técnicas cuyo objetivo es proporcionar } \\
\text { información objetiva e independiente sobre la calidad del producto a la parte interesada o stakeholder. Es una } \\
\text { actividad más en el proceso de control de calidad [16], [17]. }\end{array}$ \\
\hline \begin{tabular}{|l|} 
Software \\
Testing \\
\end{tabular} & $\begin{array}{l}\text { El proceso que consiste en todas las actividades del ciclo de vida, tanto estáticas cómo dinámicas relacionadas } \\
\text { con la planificación preparación y evaluación de productos de software y productos relacionados con el trabajo } \\
\text { para determinar que cumplen con los requisitos especificados, para demostrar que son aptos para el propósito } \\
\text { y para detectar fallos [18] }\end{array}$ \\
\hline $\begin{array}{l}\text { Prueba } \\
\text { calidad } \\
\text { software }\end{array}$ & $\begin{array}{l}\text { de El proceso de operar un sistema o un componente bajo condiciones especificadas, observando o registrando } \\
\text { de los resultados, y de hacer una evaluación de un cierto aspecto del sistema o del componente.[19] }\end{array}$ \\
\hline $\begin{array}{l}\text { Efectos de } \\
\text { prueba } \\
\text { software }\end{array}$ & oa no puede asegurar la ausencia \\
\hline
\end{tabular}

\subsubsection{FASE DOS}

La calidad del software debe tenerse en cuenta durante todo el proceso de producción de este, por lo tanto, deben existir actividades que velen por su cumplimiento durante todo el ciclo de vida, a continuación, se presenta en la figura 2 el modelo del proceso de pruebas definido.

\section{Etapas proceso}

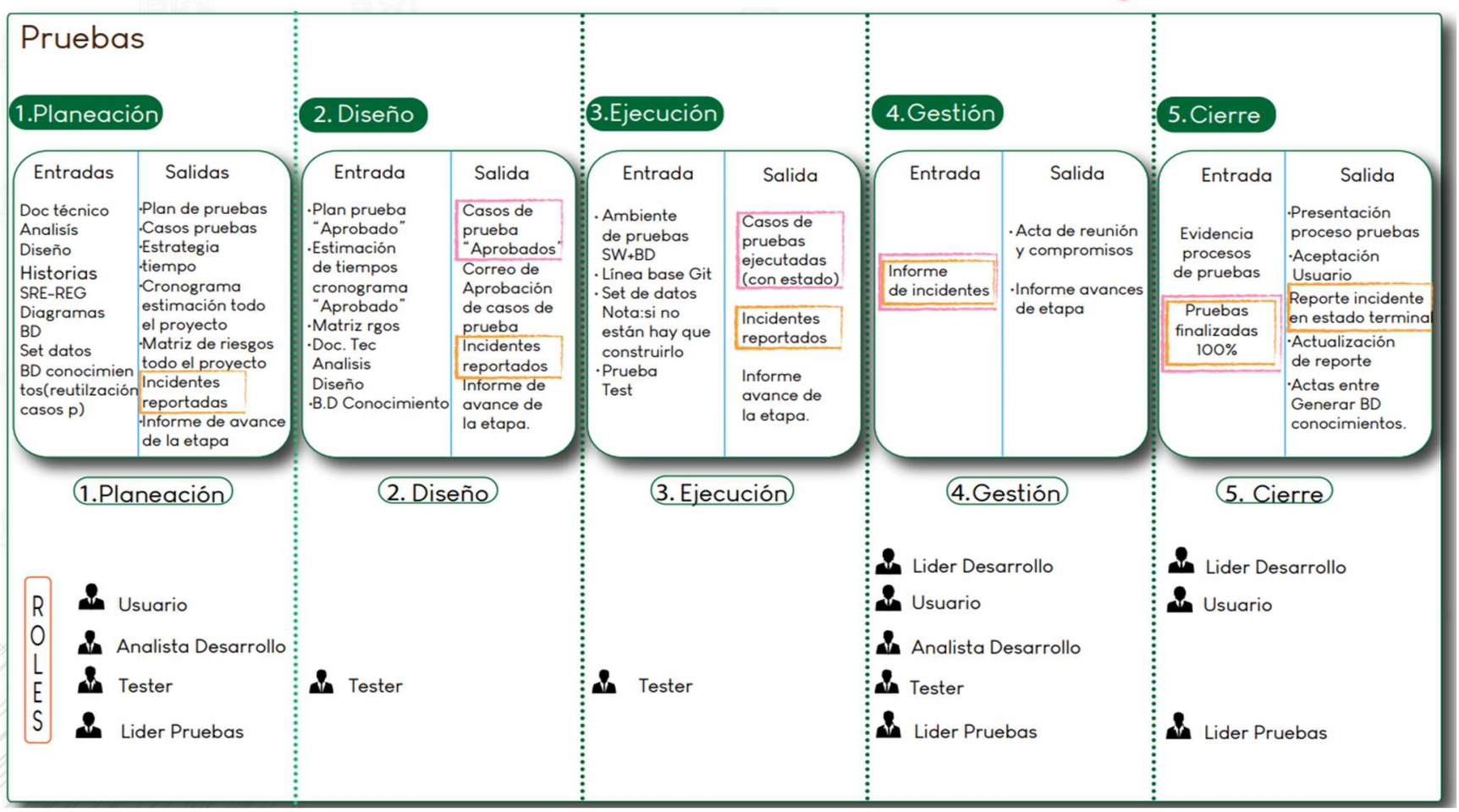

Figura. 2. Modelo de proceso de pruebas 
Propuesta de laboratorio de certificación en la norma ISO 29119 de pruebas de calidad de software en el Centro de Servicios

y Gestión Empresarial del SENA

Proposal for a certified laboratory in the iso 29119 standard for software quality tests in the SENA Center for Services and

Business Management

\subsubsection{ETAPAS DEL PROCESO DE PRUEBA}

1. Planeación: El objetivo en esta etapa es establecer las directrices, controles y mecanismos necesarios para la ejecución de las pruebas del proyecto, con el fin de asegurar el cumplimiento de los requerimientos planteados. Esta etapa comienza con la entrega de la documentación y componentes por parte del cliente, se realiza la lectura y entendimiento de la documentación, se negocia costos y recursos, así como una reunión con el usuario, analista de pruebas, líder de pruebas, para revisar los temas que no hayan quedado claros de la documentación (este paso si es que hay dudas). Se planifican las pruebas a realizar y en cada versión que se genere del producto, dicha planificación debe ser revisada ya que los supuestos sobre los que se hizo la planificación probablemente hayan cambiado. A su vez, esta etapa permite conocer el alcance de las pruebas definiendo aspectos como las entradas (requerimientos para pruebas), matriz de riesgos, estimación detallada de actividades, la estrategia de pruebas, los recursos necesarios, el cronograma y el plan de pruebas.

Responsable: Usuario, Analista de Desarrollo, Analista de Pruebas, Líder de Pruebas.

2. Diseño: El objetivo de esta etapa es el diseño de los casos de prueba del proyecto o requerimiento, a partir de los cuales se debe asegurar que se cumpla con las necesidades y expectativas del cliente. Diseñar los casos de prueba de una forma clara y concisa, siguiendo la documentación del cliente y de manera que un Analista de Pruebas los pueda ejecutar.

Responsable: Analista de Pruebas.

3. Ejecución: En esta etapa se ejecutan los casos de prueba diseñados en la etapa de "Diseño de Pruebas" con el fin de encontrar los incidentes o inconsistencias que puedan presentarse en la aplicación. Cuando se están ejecutando los casos diseñados, el Analista de Pruebas, puede detectar que hay algunos casos de prueba que no fueron tenidos en cuenta en la etapa de diseño. En este caso, se deben adicionar al formato de diseño de casos de pruebas.

Responsable: Analista de Pruebas.

4. Gestión: El objetivo de esta etapa es administrar los diferentes proyectos y recursos del proceso. Es una etapa transversal al proceso de pruebas en la cual se pretende administrar los diferentes proyectos y recursos que participen en el proceso de pruebas, igualmente, velar porque la metodología desarrollada para dicho fin se esté cumpliendo a cabalidad.

Responsable: Líder de Desarrollo, Usuario, Analista de desarrollo, Analista de Pruebas, Líder de Pruebas.

5. Cierre: Se entregan los resultados de cómo se hicieron las pruebas, cantidad de incidentes encontrados, tiempos de atraso y reprogramación, resumen de los casos de ejecutados, el alcance de cada proyecto y las estrategias definidas. Al finalizar las pruebas se realizará un paralelo de la aplicación con base a los requerimientos especificados al inicio de las pruebas.

Responsable: Líder de Desarrollo, Usuario, Líder de Pruebas.

Durante el proceso de pruebas se puede presentar situaciones en que se requiere tomar decisiones con respecto a la continuidad de las pruebas, como es el caso de devoluciones, cancelaciones, suspensiones y reanudaciones de las pruebas del proyecto.

1. Proceso de devolución: Proceso en el cual el Analista de Pruebas concluye que los artefactos a probar no cumplen con los requisitos mínimos que se necesitan para realizar el proceso. Este proceso se puede dar en cualquier etapa del ciclo de pruebas a partir de la ejecución.

Las causas de las devoluciones pueden ser:

- Parciales: Por artefactos con incidentes, aunque se puede continuar con el proceso.

- Totales: No se puede continuar con el proceso. Incidentes tipo stopper 
Razones para devolver un artefacto:

- El aplicativo no pasa el Smoke Test en la etapa de ejecución del proceso de pruebas.

- El aplicativo contiene una cantidad de errores considerables o errores de alto impacto que no permiten continuar con la prueba (Errores stopper).

- Los artefactos no son suficientes o no son claros para iniciar el proceso de pruebas.

Se debe realizar nuevamente la verificación de la funcionalidad en caso de ser una devolución total. Para las devoluciones parciales se debe validar las funcionalidades que están disponibles para ser probadas.

Se debe informar al líder de pruebas sobre las razones de la devolución. En caso de que se acepten, se envía correo al líder de desarrollo informando sobre la devolución de los artefactos para definir una fecha tentativa de la entrega de la nueva versión.

En caso de que la devolución sea total y no se pueda continuar con el proceso de pruebas, se debe solicitar un proceso de suspensión del proceso de pruebas

2. Proceso de Suspensión: Proceso en el cual el líder de pruebas solicita suspender de forma parcial o total el proceso de pruebas.

\subsubsection{Criterios de Suspensión de Pruebas}

Si por algún motivo ajeno al equipo de pruebas se presentan circunstancias que impidan la realización de las pruebas de forma fluida (ejemplo: errores, problemas de ambiente, etc.), se procederá a suspenderlas, previo manifiesto al Líder de Pruebas por medio de un correo

Las causas que ocasionarán la suspensión del proceso de prueba serán las siguientes:

- Cuando más del $20 \%$ de los defectos son reabiertos entre ciclos de pruebas.

- Cuando el ambiente de pruebas no se encuentre disponible.

- Problemas de desempeño de la máquina donde se encuentra el producto que dificultan la operación de la prueba.

- Solicitud explícita del gerente de proyectos

- Se ha presentado controles de cambios que afectan considerablemente el alcance de las pruebas.

- Retraso en entrega de artefactos

- Cambio en las prioridades de los proyectos

3. Proceso de Reanudación: Este proceso solo es válido si el proceso de pruebas se encuentra en estado Suspensión. Al reanudar un proyecto de prueba hay que verificar si el alcance ha cambiado, en caso tal se debe realizar la modificación en todas las etapas. Para reanudar formalmente la etapa de pruebas en un proyecto se debe informar al equipo de pruebas por escrito, que los inconvenientes que impedían la realización de las pruebas han sido solucionados, esto con el fin de estimar los impactos que se presentaron en los tiempos programados para las pruebas y proceder a continuar con las mismas.

4. Proceso de Cancelación: Proceso en el cual el líder de pruebas informa al equipo de pruebas la cancelación de las pruebas definitivamente.

Las causas que ocasionan cancelar las pruebas del proyecto son las siguientes:

- Por orden gerencial se cancela las pruebas a realizar, debido a que no cuenta con el recurso suficiente para ejecutarlas.

- Por decisión del cliente de cancelar las pruebas.

\subsubsection{FASE TRES}

Para tener un proceso de pruebas exitoso es necesario contar con lineamientos según la norma ISO 29119 por lo cual se define la política y estrategia organizacional.

A su vez, se definen los formatos de entrada y salida del proceso de prueba que permite el registro de la información para la trazabilidad de las pruebas. 
Propuesta de laboratorio de certificación en la norma ISO 29119 de pruebas de calidad de software en el Centro de Servicios y Gestión Empresarial del SENA

Proposal for a certified laboratory in the iso 29119 standard for software quality tests in the SENA Center for Services and Business Management

Política de pruebas: El objetivo del laboratorio de pruebas de calidad de software del Centro de Servicios y Gestión Empresarial del SENA es brindar un servicio de pruebas de software desde el inicio del ciclo de vida del proyecto, tanto para proyectos internos (Sena proveedor Sena) como externos (empresas y/o personas naturales). A su vez, desde el desarrollo de planes de prueba, la organización utilizará las actividades de prueba aplicables a proyectos desarrollados bajo metodologías ágiles y tradicional, como la participación de las partes interesadas en el diseño de la prueba, la preparación de la automatización de pruebas, revisiones por pares, diversas técnicas de diseño de pruebas (aplicable proyecto), pruebas defecto de seguimiento y presentación de informes. La política incluye objetivos del laboratorio, consideraciones importantes del proceso de pruebas y estructura, formación y ética del tester, valor de las pruebas y mejoras al proceso.

Estrategia de pruebas: En el laboratorio de pruebas de calidad de software se define como estrategia la descripción de alto nivel de los niveles de prueba que serán llevados a cabo y las pruebas dentro de estos niveles para una organización o programa (en uno o más proyectos). Teniendo como alcance definir las actividades de los proyectos y cómo estas actividades están secuenciadas y organizadas en la planificación.

- Prueba de selección y priorización: Después de analizados los requerimientos funcionales del software, se procede con la priorización de los requisitos más críticos en los cuales existe un riesgo más amplio fallo y para esto es necesario una detección temprana del error con el propósito que se realice su respectiva gestión.

- Documentación de prueba y presentación de informes: Las pruebas se documentarán desde la planeación del proyecto hasta la presentación de los indicadores generados para el proceso de pruebas.

- Gestión de incidencias: Los incidentes serán gestionados una vez sean encontrados, se crearán en la herramienta dispuesta para tal fin, donde serán asignados al desarrollador y tendrán su seguimiento.

En la tabla 2 se relacionan las plantillas definidas para el proceso de pruebas.

TABLA 2. PLANTILLAS DEL PROCESO DE PRUEBAS

\begin{tabular}{|l|l|}
\hline \multicolumn{1}{|c|}{ PLANTILLA } & \multicolumn{1}{c|}{ DEFINICIÓN } \\
\hline Acta de reunión & $\begin{array}{l}\text { Para cada reunión que se realice dentro del proceso de pruebas se debe diligenciar el } \\
\text { acta de reunión allí se plasman los temas tratados, los pendientes y las conclusiones } \\
\text { de la reunión. }\end{array}$ \\
\hline Matriz de Riesgos & $\begin{array}{l}\text { Para el proceso de pruebas se encuentra definida una matriz de riesgo, en esta matriz } \\
\text { por cada proyecto que se vaya a probar se debe diligenciar los riesgos que tiene y en } \\
\text { las reuniones de seguimiento verificar si se han materializado y así tener un plan de } \\
\text { acción por cada riesgo. }\end{array}$ \\
\hline $\begin{array}{l}\text { Estimación } \\
\text { Tiempos }\end{array}$ & $\begin{array}{l}\text { La estimación de los tiempos es uno de los subprocesos definidos ya que basados en } \\
\text { la experiencia y en el conocimiento en las pruebas se entregan los tiempos para las } \\
\text { tareas de los artefactos a probar. }\end{array}$ \\
\hline Cronograma & $\begin{array}{l}\text { El cronograma se crea cuando se entrega la estimación de los tiempos aquí podemos } \\
\text { encontrar la duración del proyecto. }\end{array}$ \\
\hline Plan de Pruebas & $\begin{array}{l}\text { En el plan de pruebas se entrega cómo se van a realizar las pruebas y que estrategias } \\
\text { se van a implementar. }\end{array}$ \\
\hline Casos de Prueba & $\begin{array}{l}\text { Esta plantilla tiene una serie de campos que al ser diligenciados tenemos como } \\
\text { producto un caso de prueba indicando sus elementos, funcionalidades y acciones a ser } \\
\text { ejercidas. }\end{array}$ \\
\hline $\begin{array}{l}\text { Aprobación } \\
\text { Casos de Prueba }\end{array}$ & $\begin{array}{l}\text { Con esta plantilla se aprueban o desaprueban los casos creados y se dan sugerencias, } \\
\text { la aprobación la debe de dar una persona diferente a la que los crea. }\end{array}$ \\
\hline $\begin{array}{l}\text { Acta de } \\
\text { Certificación }\end{array}$ & $\begin{array}{l}\text { En el acta de certificación se entrega cuando se hayan completado las pruebas para } \\
\text { todos los artefactos del proyecto teniendo en cuenta que la certificación se emite solo } \\
\text { si las pruebas se culminan exitosamente. }\end{array}$ \\
\hline $\begin{array}{l}\text { Informe Avance } \\
\text { Etapa }\end{array}$ & $\begin{array}{l}\text { Esta plantilla informa cómo culmina cada etapa, aplica para todas las etapas del } \\
\text { proceso. }\end{array}$ \\
\hline $\begin{array}{l}\text { Registro de } \\
\text { incidentes }\end{array}$ & $\begin{array}{l}\text { En esta plantilla se registran los incidentes que surgen en cada etapa del proceso e } \\
\text { indica su estado de trámite. }\end{array}$ \\
\hline $\begin{array}{l}\text { Base de datos de } \\
\text { conocimiento }\end{array}$ & $\begin{array}{l}\text { En esta plantilla se registran los casos de pruebas más comunes dependiendo el } \\
\text { proyecto. }\end{array}$ \\
\hline
\end{tabular}




\subsubsection{FASE CUATRO}

Dentro del proceso de prueba, la norma ISO 29119 enmarca las posibles técnicas de prueba que se pueden utilizar. En la tabla 3 se agrupan dichas técnicas propuestas.

TABLA 3. AGRUPACIÓN TÉCNICAS DE PRUEBA

\begin{tabular}{|l|l|}
\hline \multicolumn{1}{|c|}{ Agrupación } & \multicolumn{1}{c|}{ Técnicas } \\
\hline Técnicas de Caja Negra & $\begin{array}{l}\text { Partición de equivalencia } \\
\text { Análisis del valor límite } \\
\text { Tablas de decisión } \\
\text { Máquinas de estado finito } \\
\text { Grafo causa efecto } \\
\text { Prueba de dominios }\end{array}$ \\
\hline Técnicas de Caja Blanca & $\begin{array}{l}\text { Basadas en el flujo de control Basadas en el } \\
\text { flujo de los datos Mutantes }\end{array}$ \\
\hline $\begin{array}{l}\text { Técnicas Según Quien Hace la } \\
\text { Prueba }\end{array}$ & $\begin{array}{l}\text { Pruebas de aceptación } \\
\text { Pruebas alfa y beta } \\
\text { Pruebas de usuario } \\
\text { Pruebas en pares }\end{array}$ \\
\hline $\begin{array}{l}\text { Técnicas Basadas en la } \\
\text { Experiencia }\end{array}$ & $\begin{array}{l}\text { Prueba ad hoc } \\
\text { Conjetura de errores } \\
\text { Testing exploratorio }\end{array}$ \\
\hline
\end{tabular}

Los reportes definidos para el laboratorio de pruebas de calidad de software fueron:

1. Reporte de estado de la prueba: Permite consultar cuantos incidentes se han generado para el proyecto y la información detallada relacionada.

2. Reporte de ambientes de pruebas: Permite consultar el estado del ambiente de pruebas con las aplicaciones que en la actualidad se encuentran desplegadas para ser probadas.

3. Reporte de completitud de las pruebas: Permite consultar cuantos módulos del proyecto están certificados para ser desplegados en producción.

\section{CONCLUSIONES}

La investigación permitió realizar un análisis de la norma ISO 29119, tomando como referente las definiciones para la adecuada documentación del proceso con la que operara el laboratorio para certificar los productos de desarrollo de software con el fin de contar con una estructura organizada y gestionada para la operación del laboratorio generando resultados e informes de pruebas fomentado en aprovechamiento de las Tecnologías de la Información y las Comunicaciones en el laboratorio para la comunidad educativa en la ciudad de Medellín.

Otro punto importante es permitir el avance del estado del conocimiento de los aprendices, permitiendo la valiosa oportunidad de poner en práctica las habilidades adquiridas en formación y combinar elementos teóricos y prácticos, lo que hace que estén más motivados para el desarrollo de actividades. Así mismo, permite a los instructores fortalecer los procesos pedagógicos con el fin de mantener a los aprendices en constante aprendizaje en nuevas tecnologías.

\section{REFERENCIAS}

[1] F. J. Pino, F. Garcia, F. Ruiz, y M. Piattini, «Adaptation of the standards ISO/IEC 12207:2002 and ISO/IEC 15504:2003 for the assessment of the software processes in developing countries», IEEE Lat. Am. Trans., vol. 4, n. ${ }^{\circ}$ 2, pp. 85-92, abr. 2006, doi: 10.1109/TLA.2006.1642455.

[2] H. D. Ortiz Alzate, L. G. Muñoz Marín, J. Cardeño Espinosa, y N. C. Alzate Osorno, «Impacto del uso de objetos interactivos de aprendizaje en la apropiación de conocimiento y su contribución en el desarrollo de competencias matemáticas: un resultado de experiencia de investigación», Rev. CINTEX, vol. 21, n. ${ }^{0} 1$, pp. 7188, jun. 2016. 
Propuesta de laboratorio de certificación en la norma ISO 29119 de pruebas de calidad de software en el Centro de Servicios y Gestión Empresarial del SENA

Proposal for a certified laboratory in the iso 29119 standard for software quality tests in the SENA Center for Services and Business Management

[3] S. VELASQUEZ, J. D. VAHOS, C. LOPEZ, M. E. GOMEZ, J. LONDOÑO, y S. PATIÑO, «El rol de las normas en la acreditación y certificación de pruebas de software», Rev. Espac., vol. 39, n. ${ }^{\circ}$ 50, p. 17, 2018.

[4] A. Peralta y F. P. Romero, «Toma de Decisiones a partir de Conocimiento Extraído tras el Análisis de Comportamientos Previos. Aplicación Práctica para la Dirección de Proyectos de Desarrollo de Software», Rev. CINTEX, vol. 20, n. ${ }^{\circ}$ 2, pp. 97-111, dic. 2015.

[5] G. Salazar Bermúdez, F. Lizano Madriz, y V. Lara Villagrán, «PROPUESTA DE UN SISTEMA DE GESTION DE LA CALIDAD PARA UN LABORATORIO DE PRUEBAS», en Memorias multimedia promocional Proceedings. promotional multimedia, La Habana: CITMATEL, 2011.

[6] C. Adarve Gómez, D. A. Castillo Carvajal, E. J. Restrepo Zapata, y H. Villar-Vega, «A review of virtual reality videogames for job-training applications», Rev. CINTEX, vol. 24, n. ${ }^{\circ} 1$, pp. 64-70, dic. 2019, doi: 10.33131/24222208.346.

[7] S. Kollanus y J. Koskinen, «Survey of software inspection research», Open Softw. Eng. J., vol. 3, n. ${ }^{\circ}$, pp. 15-34, 2009, doi: 10.2174/1874107X01003010015.

[8] Y. Brito RIVEROL y T. Capote GARCÍA, «Sistema de Gestión de la Calidad en un laboratorio de evaluación de software que opta por la acreditación», Rev. Antioqueña Las Cienc. Comput. Ing. Softw. RACCIS, vol. 3, n. ${ }^{\circ}$ 2, pp. 13-18, 2013.

[9] J. C. Morales Saldarriaga, K. Fernández Morales, y J. E. Pulido, «Evaluación de técnicas de producción accesible en cursos masivos, abiertos y en línea - MOOC», Rev. CINTEX, vol. 21, n. 1, pp. 89-112, 2016.

[10] C. Garcia, A. Dávila, y M. Pessoa, «Test Process Models: Systematic Literature Review», en Software Process Improvement and Capability Determination, vol. 477, A. Mitasiunas, T. Rout, R. V. O'Connor, y A. Dorling, Eds. Cham: Springer International Publishing, 2014, pp. 84-93.

[11] J. J. Castro-Maldonado, J. A. Londoño-Gallego, S. Londoño-Marin, y J. A. Patiño-Murillo, «lmplementation of a technological, information, and communication tool for project management in the network of Tecnoparque, Colombia», J. Phys. Conf. Ser., vol. 1418, p. 012014, dic. 2019, doi: 10.1088/1742-6596/1418/1/012014.

[12] M. I. Ardila Marín, W. Orozco Murillo, J. Galeano Echeverri, y A. M. Medina Escobar, «Desarrollo de software para la gestión del mantenimiento en los laboratorios de la I.U. Pascual Bravo», Rev. CINTEX, vol. 23, n. ${ }^{\circ}$, pp. 43-50, oct. 2018.

[13] J. D. Vahos, A. A. Pino, y J. J. Castro Maldonado, «Desarrollo de una herramienta de software para la gestión del mantenimiento de infraestructura en el SENA regional Antioquia», Rev. CINTEX, vol. 24, n. ${ }^{\circ 1}$, pp. 1319, dic. 2019, doi: 10.33131/24222208.331.

[14] M. Fandos Garrido, Á. P. González Soto, Universitat Rovira i Virgili, Departament de Pedagogia, Universitat Rovira i Virgili, y Facultat de Ciències de l'Educació i Psicologia de Tarragona, Formación basada en las tecnologías de la información y la comunicación análisis didáctico del proceso de enseñanza - aprendizaje: tesis doctoral. Tarragona: Universitat Rovira i Virgili, 2005.

[15] R. Eito-Brun y M.-A. Sicilia, «An innovation activity model for Very Small Entities in the software sector: an empirical study: Innovation activity model in the software sector», RD Manag., vol. 47, n. ${ }^{\circ}$ 5, pp. E13-E25, nov. 2017, doi: 10.1111/radm.12226.

[16] G. Pantaleo, Calidad en el desarrollo de software. México; Barcelona: Alfaomega; Marcombo, 2012.

[17] J. Echeverri, M. Aristizábal, y L. González, Reflexiones sobre ingeniería de requisitos y pruebas de software. Medellín: Corporación Universitaria Remington, 2013.

[18] O. A. Pérez A., "Cuatro enfoques metodológicos para el desarrollo de Software RUP - MSF - XP SCRUM», INVENTUM, vol. 6, n. ${ }^{\circ}$ 10, pp. 64-78, feb. 2011, doi: 10.26620/uniminuto.inventum.6.10.2011.64-78.

[19] E. A. Cáceres, "Análisis y diseño de sistemas de información», Universidad Nacional de San Juan, Rivadavia - San Juan, Argentina, Notas de curso, 2014.

[20] M. Callejas-Cuervo, A. C. Alarcón-Aldana, y A. M. Álvarez-Carreño, «Modelos de calidad del software, un estado del arte», ENTRAMADO, vol. 13, n. ${ }^{\circ}$, pp. 236-250, 2017, doi: 10.18041/entramado.2017v13n1.25125. 